\title{
Orthogonal cross-task compatibility: Abstract spatial coding in dual tasks
}

\author{
IRING KOCH \\ Max Planck Institute of Human Cognitive and Brain Sciences, Leipzig, Germany \\ and RWTH Aachen University, Aachen, Germany \\ AND \\ Pierre Jolichur \\ Université de Montréal, Montreal, Quebec, Canada
}

\begin{abstract}
Spatially orthogonal stimulus and response sets can produce compatibility effects. To explore whether such effects cross the border of logically independent tasks, we combined a nonspeeded visual task requiring verbal report of a stimulus movement (up vs. down) with an auditory reaction time task that required a unimanual movement to the left or right. Two experiments demonstrated that $u p$ stimuli facilitate rightward responses and down stimuli facilitate leftward responses, relative to the opposite combinations, thus producing an orthogonal cross-task compatibility effect. This effect presumably arises from abstract coding with respect to the salient referents of a spatial dimension (i.e., up and right), so that coactivation of structurally similar codes leads to mutual priming even when the codes refer to different tasks. The present evidence for abstract spatial coding extends previously proposed coding principles from single-task settings to dual-task settings.
\end{abstract}

Action selection is strongly determined by the stimulus characteristics of a situation. For instance, left or right keypresses are faster when the required action is assigned to stimuli from the left or right, respectively, producing the phenomenon of stimulus-response (S-R) compatibility. There is evidence that relatively abstract cognitive coding processes contribute to S-R compatibility (see, e.g., Lien \& Proctor, 2002, for a review). The abstractness of cognitive coding can perhaps be best exemplified with the phenomenon of orthogonal S-R compatibility (see Cho $\&$ Proctor, 2003, for a review).

Orthogonal S-R compatibility designates the finding that there are compatibility relations between vertical stimulus positions and horizontal response positions, so that the spatial stimulus and response sets are aligned along orthogonal, rather than parallel, directions. With orthogonal stimulus and response sets, there is a general advantage of the up-right/down-left mapping over the up-left/down-right mapping (e.g., Bauer \& Miller, 1982; Weeks \& Proctor, 1990). To account for orthogonal S-R compatibility, Weeks and Proctor advanced the salientfeatures-coding hypothesis, stating that $u p$ and right are the salient polar referents for their respective spatial dimension and that $\mathrm{S}-\mathrm{R}$ translation is faster when the salience structure of the stimulus set corresponds to that of the response set (Cho \& Proctor, 2003).

In the present study, we examined whether orthogonal S-R compatibility also crosses the borders of logically independent tasks, giving rise to orthogonal cross-task compatibility. The experimental paradigm combined a nonspeeded visual task with a speeded auditory task. In the visual task, subjects encoded an upward or downward moving dot for deferred verbal report. The dot was presented briefly and was then masked to enforce immediate encoding into short-term memory. After some stimulus onset asynchrony (SOA), the dot was followed by a tone. In the auditory task, the subjects had to perform a speeded two-choice pitch discrimination response (move a finger left or right). We tested whether an upward visual stimulus movement (in the visual task) facilitated a rightward manual response (in the tone task) and a downward movement a leftward response, relative to the opposite combinations. This finding would constitute an orthogonal cross-task compatibility effect. Importantly, although there are studies showing effects of orthogonal S-R compatibility (e.g., Cho \& Proctor, 2003), as well as studies showing parallel cross-task compatibility (e.g., Koch \& Prinz, 2002; Lien $\&$ Proctor, 2002), there has been no study examining orthogonal cross-task compatibility.

Finding an orthogonal cross-task compatibility effect would imply that concurrent activation of codes referring to orthogonal spatial dimensions can lead to mutual priming even when these codes refer to entirely different tasks. Such a finding would support the notion of abstract, central codes and would generalize the salient-features-coding account from single-task settings to dual-task settings.

In orthogonal S-R compatibility, there is always close temporal proximity of stimulus presentation and response

I. Koch, koch@psych.rwth-aachen.de 
selection because both refer to the same task. In contrast, in orthogonal cross-task compatibility, the $u p$ versus down code is relevant for the visual task, but not for the auditory task. We manipulated the SOA between visual stimulus and tone to explore whether orthogonal crosstask compatibility would depend on temporal proximity of encoding in the visual task and response selection in the auditory task. With a short SOA, the cognitive code referring to the stimulus movement would be activated shortly before response selection to the tone, so we predicted stronger response priming in the auditory task with a short SOA than with a long SOA.

Previous research has shown that visual encoding for later report requires a process that consolidates the code in short-term memory. This consolidation process may interfere with concurrent response selection in the second task (e.g., Jolicœur \& Dell'Acqua, 1998; Koch, Metin, \& Schuch, 2003; Koch \& Prinz, 2002, 2005). That is, in addition to establishing the phenomenon of orthogonal cross-task compatibility, we expected to observe generally impaired performance with a short SOA, relative to a long SOA, which would support the idea that memory processes can create cognitive bottlenecks in dual-task performance (for reviews, see Jolicœur, Tombu, Oriet, \& Stevanovski, 2002; Pashler, 2000).

\section{EXPERIMENT 1}

\section{Method}

Subjects. Sixteen adult subjects participated for $€ 7.5$.

Apparatus and Stimuli. Manual responses were performed on an external keyboard with three keys (1.8- $\mathrm{cm}$ width, $3.3 \mathrm{~cm}$ apart). Responses were executed with the index finger of the dominant hand. The middle key, centered at body midline and with respect to the screen, was a home key that had to be kept pressed at the beginning of each trial. The response signal was a high $(900-\mathrm{Hz})$ or low $(300-\mathrm{Hz})$ tone presented for $50 \mathrm{msec}$, calling for a finger movement to either the left or the right key. Response time (RT) was measured as the latency between tone onset and pressing the specified key. The tone was emitted by the internal speaker of the PC, placed in front of the subjects. The visual stimulus was a black dot $(0.2 \mathrm{~cm})$ displayed for $17 \mathrm{msec}$ at the center of a 15 -in. VGA screen, then for another $17 \mathrm{msec}$ at a position $0.2 \mathrm{~cm}$ above or below, and finally for $17 \mathrm{msec}$ at a position $0.4 \mathrm{~cm}$ above or below screen center, creating an impression of upward or downward motion. The dot was followed by a mask $(5.6 \times 9.7 \mathrm{~cm})$, with pixels set at random.

Procedure. The subjects were seated in front of the screen. Task instructions appeared on the screen but were also given verbally. In the visual task, the subjects had to report the movement direction of the target stimulus (S1) at the end of the trial by saying "up" or "down" (in German "oben" and "unten"). The experimenter recorded the verbal response (R1), using the corresponding arrow keys from the standard keyboard. In the auditory task, the subjects heard a low-pitched $(300-\mathrm{Hz})$ or a high-pitched $(900-\mathrm{Hz})$ tone for $50 \mathrm{msec}$ (S2). The tone was presented at a variable SOA (100 vs. 1,200 msec) with respect to the moving dot. The response to the tone was made with the index finger of the dominant hand (R2). The tone-to-key (S2-R2) assignment was counterbalanced across subjects.

The subjects performed a practice block of 32 trials to become familiar with the two tasks. They then performed six blocks of 64 trials each. Between blocks, a message was displayed offering an optional rest period and informing the subjects how many blocks were yet to come. Each trial commenced with a message on the screen reminding the subjects of the tone-to-key mapping (although the mapping remained constant for a given subject) and that they had to press the home key to start the next trial, keeping this key pressed until tone onset. Pressing the home key triggered, after $500 \mathrm{msec}$, the presentation of the visual target, followed immediately by the mask and, after a variable SOA, the tone for the auditory task. The mask remained on the screen until execution of the response in the tone task. The correct sequence of responses was to release the home key and press the required left or right key, followed by the nonspeeded verbal response in the visual task. Releasing the home key too early or pressing the wrong key was scored as an error. Feedback on errors in the auditory task was a 500-msec display of the German word "falsch" (wrong) at screen center. After response execution or error feedback, another $500 \mathrm{msec}$ elapsed before the next written message appeared, asking whether the apparent movement on the screen had been upward or downward, followed by feedback on accuracy for this task.

Design. Orthogonal cross-task compatibility (compatible vs. incompatible) and SOA (100 vs. 1,200 msec) were manipulated independently as within-subjects variables. Compatibility was defined on the basis of the $\mathrm{S} 1-\mathrm{R} 2$ relation. A trial was considered compatible when an upward stimulus movement co-occurred with a rightward response or when a downward movement co-occurred with a leftward response; the other trials were considered incompatible. The dependent measures were RT and proportion correct for the auditory RT task and the visual task. All significance tests were conducted with an alpha level of .05 .

\section{Results and Discussion}

Visual task. Table 1 shows the results of Experiment 1. For the visual task, we first discarded trials with an error in the auditory task. We ran an ANOVA for proportion correct, with the within-subjects independent variables of compatibility (compatible vs. incompatible) and SOA (100 vs. 1,200 $\mathrm{msec}$ ), which yielded a significant effect of compatibility $\left[F(1,15)=9.680, M S_{\mathrm{e}}=0.002, p<.01\right]$, indicating higher accuracy for compatible than for incompatible trials (.899 vs. .864) and, thus, a compatibility effect of .035 . The effect of SOA $\left[F(1,15)=1.657, M S_{\mathrm{e}}=\right.$ $0.001, p>.21]$ was not significant, unlike the interaction $\left[F(1,15)=5.720, M S_{\mathrm{e}}=0.000, p<.05\right]$. The interaction was due to a stronger compatibility effect with a short SOA (.049) than with a long SOA (.022).

Auditory task. We discarded trials with errors in the visual task. We then submitted proportion correct to an

Table 1

Experiment 1: Performance in the Visual Task and in the Auditory Task as a Function of Stimulus Onset Asynchrony (SOA) and Cross-Task Compatibility

\begin{tabular}{|c|c|c|c|c|}
\hline & \multicolumn{4}{|c|}{$\mathrm{SOA}(\mathrm{msec})$} \\
\hline & \multicolumn{2}{|c|}{100} & \multicolumn{2}{|c|}{1,200} \\
\hline & $M$ & $S D$ & $M$ & $S D$ \\
\hline \multicolumn{5}{|c|}{ Visual Task (Proportion Correct) } \\
\hline Incompatible & .852 & .159 & .875 & .148 \\
\hline Compatible & .900 & .135 & .897 & .137 \\
\hline \multicolumn{5}{|c|}{ Auditory Task (Proportion Correct) } \\
\hline Incompatible & .931 & .058 & .957 & .035 \\
\hline Compatible & .975 & .029 & .973 & .024 \\
\hline \multicolumn{5}{|c|}{ Auditory Task (Response Time, in msec) } \\
\hline Incompatible & 991 & 397 & 719 & 256 \\
\hline Compatible & 924 & 361 & 695 & 238 \\
\hline
\end{tabular}


ANOVA with compatibility and SOA as independent variables. Proportion correct was higher for compatible than for incompatible trials $[.974$ vs. .944; $F(1,15)=13.727$, $\left.M S_{\mathrm{e}}=0.001, p<.01\right]$. The effect of SOA was nonsignificant $\left[F(1,15)=2.584, M S_{\mathrm{e}}=0.001, p>.125\right]$, but the interaction was significant $\left[F(1,15)=5.091, M S_{\mathrm{e}}=\right.$ $0.001, p<.05]$. The interaction was due to the fact that the compatibility effect was larger with the short SOA [.044; $t(15)=3.832, p<.01$, when tested separately] than with the long SOA [.016; $t(15)=1.837, p<.05$, one-tailed].

For RT analysis, we discarded trials with an error in either task or both tasks and dropped as outliers trials with RTs above 3,000 msec ( $<1 \%)$. The ANOVA yielded significant main effects of compatibility $[F(1,15)=23.048$, $\left.M S_{\mathrm{e}}=1,427, p<.001\right]$ and SOA $[F(1,15)=42.561$, $\left.M S_{\mathrm{e}}=23,666, p<.001\right]$. RT for incompatible trials was higher than that for compatible trials ( $855 \mathrm{vs} .810 \mathrm{msec})$. RT was higher at the short SOA than at the long SOA (958 vs. $707 \mathrm{msec})$. Also, the interaction was significant $\left[F(1,15)=12.487, M S_{\mathrm{e}}=601, p<.001\right]$, indicating that the compatibility effect was larger at the short SOA $[67 \mathrm{msec} ; t(15)=4.870, p<.001$, when tested separately] than at the long SOA [24 $\mathrm{msec} ; t(15)=2.955$, $p<.01]$.

In sum, the results show a clear orthogonal cross-task compatibility effect. This effect decreased with increases in SOA but was reliable even with an SOA of 1,200 msec. The decrease suggests that cross-task compatibility is primarily due to cross-talk during the encoding process. The residual effect at the long SOA might still be caused by the encoding process, which might not have been completed after a long SOA on some trials, or to maintenance of the memorized code. We also found dual-task interference, measured as the SOA main effect. A higher RT with a short SOA indicates that visual encoding into short-term memory impaired action selection in the other task, supporting previous findings (e.g., Jolicœur et al., 2002; Koch \& Prinz, 2002).

\section{EXPERIMENT 2}

The aim of Experiment 2 was to replicate the orthogonal cross-task compatibility effect and to examine the role of stimulus relevance for the effect by introducing an instruction manipulation. Prior to some blocks of trials, the subjects were instructed to ignore the visual stimulus and to perform only the auditory task. However, in the interpretation of the data, we will focus on the orthogonal cross-task compatibility effect obtained in those blocks that exactly replicated the conditions in Experiment 1.

\footnotetext{
Method

Subjects. Sixteen new adult subjects participated for $€ 7.5$.

Apparatus, Stimuli, Procedure, and Design. The apparatus, stimuli, procedure, and design were the same as those in Experiment 1 , except that we included an instruction manipulation as a third within-subjects variable. In half of the experimental blocks, the subjects were instructed to ignore the visual stimulus and simply perform the auditory task. The visual stimulus was presented nevertheless in each trial. The task instruction was given by a message prior to each block. The subjects first received 16 practice trials,
}

followed by six experimental blocks of 64 trials each. Report and ignore blocks alternated, with block order counterbalanced across subjects.

\section{Results and Discussion}

Visual task. The results of Experiment 2 are shown in Table 2. For proportion correct, the ANOVA with the independent variables of compatibility and SOA yielded no significant effects $\left[F(1,15)=1.651, M S_{\mathrm{e}}=0.001\right.$, $p>.21$, for the effect of SOA; other Fs $<1]$. Note that proportion correct was higher in Experiment 2 than in Experiment 1 (.930 vs. .881$)$, but this difference was not significant when experiment was included as an additional variable $\left[F(1,30)=1.572, M S_{\mathrm{e}}=0.048, p>.21\right]$.

Auditory task. For proportion correct, we ran an ANOVA with the within-subjects variables of compatibility, SOA, and instruction (report vs. ignore). There was a significant effect of instruction $[F(1,15)=11.343$, $\left.M S_{\mathrm{e}}=0.000, p<.01\right]$. Proportion correct was higher in ignore trials (.982) than in report trials (.971), suggesting that dual-task requirements interfered with accuracy of manual response execution. No other effect was significant $(F \mathrm{~s}<1)$.

For the RT analysis, we excluded errors and outliers (RT $>3,000 \mathrm{msec} ;<1 \%$ ) and then ran the same type of ANOVA, which yielded significant effects of compatibility, SOA, and instruction. RT was generally higher on incompatible than on compatible trials [756 vs. $745 \mathrm{msec}$; $\left.F(1,15)=5.646, M S_{\mathrm{e}}=642, p<.05\right]$, higher at the short SOA than at the long SOA [821 vs. $679 \mathrm{msec} ; F(1,15)=$ $\left.125.553, M S_{\mathrm{e}}=5,154, p<.01\right]$, and also higher in report than in ignore blocks [861 vs. $639 \mathrm{msec} ; F(1,15)=$ $\left.39.438, M S_{\mathrm{e}}=40,051, p<.01\right]$. However, both the compatibility variable and the SOA variable interacted sig-

Table 2

Experiment 2: Performance in the Visual Task and in the Auditory Task as a Function of Stimulus Onset Asynchrony (SOA) and Cross-Task Compatibility, and the Report Versus Ignore Conditions for the Auditory Task

\begin{tabular}{|c|c|c|c|c|}
\hline \multirow[b]{3}{*}{ Condition } & \multicolumn{4}{|c|}{ SOA (msec) } \\
\hline & \multicolumn{2}{|c|}{100} & \multicolumn{2}{|c|}{1,200} \\
\hline & $M$ & $S D$ & $M$ & $S D$ \\
\hline \multicolumn{5}{|c|}{ Visual Task (Proportion Correct) } \\
\hline Incompatible & .914 & .099 & .938 & .067 \\
\hline Compatible & .933 & .044 & .934 & .070 \\
\hline \multicolumn{5}{|c|}{ Auditory Task (Proportion Correct) } \\
\hline \multicolumn{5}{|l|}{ Report } \\
\hline Incompatible & .968 & .029 & .971 & .032 \\
\hline Compatible & .974 & .043 & .970 & .028 \\
\hline \multicolumn{5}{|l|}{ Ignore } \\
\hline Incompatible & .986 & .024 & .977 & .037 \\
\hline Compatible & .982 & .020 & .983 & .020 \\
\hline \multicolumn{5}{|c|}{ Auditory Task (Response Time, in msec) } \\
\hline \multicolumn{5}{|l|}{ Report } \\
\hline Incompatible & 1,008 & 239 & 743 & 191 \\
\hline Compatible & 975 & 246 & 719 & 174 \\
\hline \multicolumn{5}{|l|}{ Ignore } \\
\hline Incompatible & 650 & 112 & 621 & 104 \\
\hline Compatible & 652 & 115 & 633 & 110 \\
\hline
\end{tabular}


nificantly with the encoding manipulation. The compatibility effect was larger in report than in ignore blocks $\left[29\right.$ vs. $-7 \mathrm{msec} ; F(1,15)=9.429, M S_{\mathrm{e}}=1,075, p<$ $.01]$, and also, the SOA effect was larger in report than in ignore blocks [ 260 vs. $21 \mathrm{msec} ; F(1,15)=73.136, M S_{\mathrm{e}}=$ $6,071, p<.01]$. The interaction of compatibility and SOA $[F(1,15)=1.410, p>.25]$ and the three-way interaction $(F<1)$ were nonsignificant.

When the compatibility effect was tested separately for each SOA level in the report blocks, it was significant at both SOAs $[33 \mathrm{msec}, t(15)=2.682, p<.05$, and $24 \mathrm{msec}, t(15)=2.308, p<.05$, for short and long SOAs, respectively]. In ignore blocks, the compatibility effect was not significant at an SOA of $100 \mathrm{msec}(-2 \mathrm{msec}$, $p>.8)$ or at an SOA of $1,200 \mathrm{msec}[-12 \mathrm{msec} ; t(15)=$ $-1.780, p>.095]$.

In sum, the RT results confirmed the existence of orthogonal cross-task compatibility. As in Experiment 1, the compatibility effect decreased with the long SOA in the report blocks, but this decrease was numerically less pronounced than in Experiment 1. Also, the compatibility effect at the short SOA was numerically smaller in Experiment 2 than in Experiment 1 (i.e., 33 vs. $67 \mathrm{msec}$ ). However, when we ran a mixed ANOVA to compare RT in both experiments, using only the report blocks in Experiment 2 , we found significant effects of compatibility and SOA (both $F \mathrm{~s}>32, p \mathrm{~s}<.001$ ), as well as a significant interaction of both $\left[F(1,30)=8.051, M S_{\mathrm{e}}=693, p<.01\right]$, but the three-way interaction of compatibility, SOA, and experiment was not significant $\left[F(1,30)=3.300, M S_{\mathrm{e}}=\right.$ $693, p<.079]$. The experiment variable also did not produce any other significant effects (all $p s>.2$ ).

The complete disappearance of the compatibility effect in the ignore blocks suggests that orthogonal cross-task compatibility is less automatic than parallel cross-task compatibility, where significant effects have been observed also in ignore conditions (e.g., Koch et al., 2003). Thus, explicit coding of the up-down dimension, even if it occurs in the context of an unrelated task, appears to be necessary for orthogonal cross-task compatibility effects to occur. This difference between orthogonal and parallel cross-task compatibility would be an interesting issue for future experiments.

\section{GENERAL DISCUSSION}

Two experiments demonstrated orthogonal cross-task compatibility. Importantly, compatibility in the present context does not refer to stimuli and responses within a task, as in orthogonal S-R compatibility, but rather, to compatibility across tasks. We also observed substantial SOA effects, suggesting that encoding a visual stimulus into short-term memory postpones concurrent stimulusbased response selection (Jolicœur \& Dell'Acqua, 1998; Jolicœur et al., 2002; Koch \& Prinz, 2002). Hence, the present memory requirements produced a process bottleneck similar to that suggested to account for dual-task interference with two speeded tasks (see, e.g., Jolicœur et al., 2002; Koch \& Prinz, 2005; Pashler, 2000).
To account for the finding of an orthogonal cross-task compatibility effect, we assume that it arises from abstract coding with respect to the salient referents of a spatial dimension (i.e., up and right), so that coactivation of similar codes leads to priming even when the codes refer to different tasks. That is, our account generalizes the salientfeatures-coding hypothesis advanced by Weeks and Proctor (1990) from coding that is restricted to one task to coding in dual tasks. Note that the present orthogonal compatibility effect was numerically at least as large as that in single-task studies (see Cho \& Proctor, 2003, for a review). Our findings imply pragmatically that orthogonal spatial dimensions cannot be used as neutral baseline conditions for each other in dual-task settings. At the level of theory, the implication is that the salient-features-coding account is applicable to code interactions across tasks.

Note that our results show clear evidence of orthogonal cross-task compatibility, but our auditory task might also have produced orthogonal $S 2-R 2$ within-task compatibility. Specifically, we mapped high versus low tones to right versus left manual responses. It has been reported that the coding of pitch is related to coding of space (i.e., up vs. down) and, thus, might produce effects of vertically parallel S-R compatibility (e.g., Mudd, 1963). Our design mapped high and low tones to left and right responses, so that we can ask whether there was an orthogonal S2-R2 compatibility effect (i.e., an advantage of the high-right/ low-left assignment over the other assignment). Because the $\mathrm{S}-\mathrm{R}$ assignment was counterbalanced across subjects in both experiments, we tested for orthogonal S-R compatibility in the auditory task by collapsing the data from both experiments (taking only the report blocks in Experiment 2) and reanalyzing them with the additional variable of group (i.e., S2-R2 mapping). However, there was no evidence for an orthogonal S2-R2 compatibility effect in RT (RT was even slightly longer in the group with compatible S2-R2 mapping) or accuracy in the auditory task $(F \mathrm{~S}<1)$. This absence of an orthogonal S2-R2 compatibility effect in the auditory task might have been due to the indirectness of the relation between pitch and space.

However, considering the (counterbalanced) S2-R2 mapping allows an additional analysis that helps to clarify the theoretically important question as to the nature of the codes that mediated the obtained orthogonal crosstask compatibility effects. In our paradigm, there were four possible orthogonal cross-task compatibility relations: S1-S2, S1-R2, S2-R1, and R1-R2. In principle, any of these relations could have been effective. When the S2-R2 mapping in the auditory task is compatible (on the basis of theoretical considerations; see above), all four relations coincide completely (see Table 3). However, the incompatible $\mathrm{S} 2-\mathrm{R} 2$ mapping inverted two relations (i.e., $\mathrm{S} 1-\mathrm{R} 2$ and R1-R2).

Recall that we defined a trial as being cross-task compatible on the basis of the $\mathrm{S} 1-\mathrm{R} 2$ relation (see the Design section in Experiment 1). Hence, comparing the orthogonal cross-task compatibility effect in the two S2-R2 mapping groups would enable us to draw conclusions as regards the underlying functionally effective compatibility 
Table 3

Overview of Experimental Conditions and Resulting Compatibility Relations Across Tasks

\begin{tabular}{|c|c|c|c|c|c|c|c|}
\hline $\begin{array}{l}\text { Visual Movement } \\
\text { Stimulus (S1) }\end{array}$ & $\begin{array}{l}\text { Auditory Tone } \\
\text { Stimulus (S2) }\end{array}$ & $\begin{array}{c}\text { Speeded Manual } \\
\text { Response (R2) }\end{array}$ & $\begin{array}{c}\text { Nonspeeded Vocal } \\
\text { Response (R1) }\end{array}$ & $\mathrm{S} 1-\mathrm{S} 2$ & $\mathrm{~S} 1-\mathrm{R} 2$ & $\mathrm{~S} 2-\mathrm{R} 1$ & $\mathrm{R} 1-\mathrm{R} 2$ \\
\hline \multicolumn{8}{|c|}{ Compatible S2-R2 Mapping in the Auditory Task } \\
\hline Up & high & right & up & + & + & + & + \\
\hline Up & low & left & up & - & - & - & - \\
\hline Down & high & right & down & - & - & - & - \\
\hline Down & low & left & down & + & + & + & + \\
\hline \multicolumn{8}{|c|}{ Incompatible S2-R2 Mapping in the Auditory Task } \\
\hline Up & high & left & up & + & - & + & - \\
\hline Up & low & right & up & - & + & - & + \\
\hline Down & high & left & down & - & + & - & + \\
\hline Down & low & right & down & + & - & + & - \\
\hline
\end{tabular}

Note- “+" = compatible relation across tasks. "-" = incompatible relation across tasks. Compatibility of S2-R2 mapping refers to orthogonal S-R (pitch-space) compatibility in the auditory task.

relation. If orthogonal cross-task compatibility had been exclusively due to $\mathrm{S} 1-\mathrm{S} 2$ or $\mathrm{S} 2-\mathrm{R} 1$ relations, the effect should be completely reversed in the incompatible $\mathrm{S}-\mathrm{R}$ mapping group, relative to the other group, because the S1-S2 compatible trials would be coded as incompatible on the basis of the S1-R2 relation. If so, however, when collapsing the groups, as we did in the original analysis, the effect would have been canceled out, to result in a net effect of zero. This was apparently not the case.

In fact, the above-mentioned ANOVA for the RT data showed significant effects of SOA, cross-task compatibility, and a significant interaction of both, but, importantly, it also revealed a significant interaction of cross-task compatibility and group $\left[F(1,30)=4.677, M S_{\mathrm{e}}=1,238, p<\right.$ $.05]$. This interaction indicated that the compatibility effect was larger in the compatible S2-R2 mapping group than in the incompatible mapping group (50 vs. $23 \mathrm{msec}$ ). When tested separately, the 23-msec effect was still significant in the latter group $\left[F(1,15)=9.435, M S_{\mathrm{e}}=\right.$ 932, $p<.01]$. (All other effects were nonsignificant in this ANOVA; $p \mathrm{~s}>$.18.) The same pattern of results was obtained also for the accuracy data in the auditory task $\left[F(1,30)=4.542, M S_{\mathrm{e}}=0.001, p<.05\right.$, for the interaction of compatibility and group], whereas this interaction was nonsignificant in the visual task $(F<1)$.

There are two possible explanations for the numerical decrease of the size of the cross-task compatibility effect in the group with the incompatible S2-R2 mapping, relative to the other group. First, it is possible that all relations contribute directly and independently to the observed compatibility effect (Ben-Artzi \& Marks, 1999) but that S1-S2 and S2-R1 relations have a relatively smaller weight than does the $\mathrm{S} 1-\mathrm{R} 2$ or the $\mathrm{R} 1-\mathrm{R} 2$ relation. If so, the net effect should be reduced but not eliminated when $\mathrm{S} 1-\mathrm{S} 2$ and $\mathrm{S} 2-\mathrm{R} 1$ relations are incompatible, just as was observed. Alternatively, it is possible that orthogonal cross-task compatibility is produced mainly by $\mathrm{S} 1-\mathrm{R} 2$ or R1-R2 relations. Then, there would be only an indirect contribution of the S1-S2 or S2-R1 relation, due to the fact that in the group with incompatible S2-R2 mapping, these relations were not congruent with the $\mathrm{S} 1-\mathrm{R} 2$ and $\mathrm{R} 1-\mathrm{R} 2$ relations. This would imply that these relations were strong enough to influence performance but that they exert this influence only indirectly by modulating the influence of the functionally effective $\mathrm{S} 1-\mathrm{R} 2$ or R1-R2 relation. Note that, with respect to $\mathrm{R} 1-\mathrm{R} 2$ relations, $\mathrm{R} 1$ refers to the visual stimulus. Because masking of this stimulus most likely enforced immediate encoding, it is likely that this resulted in a verbal code (i.e., R1), making the distinction between the code referring to $\mathrm{S} 1$ and that referring to $\mathrm{R} 1$ difficult. Therefore, the present data do not allow a clear differentiation of $\mathrm{S} 1-\mathrm{R} 2$ and $\mathrm{R} 1-\mathrm{R} 2$ relations.

The issue of whether the $\mathrm{S} 1-\mathrm{R} 2$ or the $\mathrm{R} 1-\mathrm{R} 2$ relation is relatively more important for dual-task code interactions was recently directly addressed in a study that examined parallel cross-task compatibility, using precued manual responses (Koch \& Prinz, 2005). The visual task in that study required subjects to respond to a leftward moving stimulus by saying "right" (and vice versa), so that the $\mathrm{S} 1-\mathrm{R} 2$ relation was incompatible when the $\mathrm{R} 1-\mathrm{R} 2$ relation was compatible. The results suggested that parallel cross-task compatibility was due to the $\mathrm{R} 1-\mathrm{R} 2$ relation. It seems likely that the same would be true for the present paradigm, but given that the present effect is a different kind of cross-task compatibility effect, explicit studies designed to show this would be required to close the issue.

In sum, the present study demonstrated orthogonal cross-task compatibility, which allows us to extend our knowledge of coding principles based on singletask performance (Cho \& Proctor, 2003) to dual-task performance.

\section{AUTHOR NOTE}

The authors thank Anne P. Hillstrom, Robert Proctor, Eric Ruthruff, and two anonymous reviewers for helpful comments on a previous version of the manuscript. Correspondence concerning this article should be addressed to I. Koch, Institute of Psychology, RWTH Aachen University, Jägerstr. 17-19, D-52056 Aachen, Germany (e-mail: koch@psych .rwth-aachen.de).

\section{REFERENCES}

Bauer, D. W., \& Miller, J. (1982). Stimulus-response compatibility and the motor system. Quarterly Journal of Experimental Psychology, 34A, 367-380.

Ben-Artzi, E., \& Marks, L. E. (1999). Processing linguistic and per- 
ceptual dimensions of speech: Interactions in speeded classifications. Journal of Experimental Psychology: Human Perception \& Performance, 25, 579-595.

Cho, Y. S., \& Proctor, R. W. (2003). Stimulus and response representations underlying orthogonal stimulus-response compatibility effects. Psychonomic Bulletin \& Review, 10, 45-73.

Joliceeur, P., \& Dell'ACQuA, R. (1998). The demonstration of shortterm consolidation. Cognitive Psychology, 36, 138-202.

Joliceeur, P., Tombu, M., Oriet, C., \& Stevanovski, B. (2002). From perception to action: Making the connection. In W. Prinz \& B. Hommel (Eds.), Attention and performance XIX: Common mechanisms in perception and action (pp. 558-586). Oxford: Oxford University Press.

Koch, I., Metin, B., \& ScHUCH, S. (2003). The role of temporal unpredictability for process interference and code overlap in perceptionaction dual tasks. Psychological Research, 67, 244-252.

Koch, I., \& Prinz, W. (2002). Process interference and code overlap in dual-task performance. Journal of Experimental Psychology: Human Perception \& Performance, 28, 192-201.
Koch, I., \& PrInZ, W. (2005). Response preparation and code overlap in dual tasks. Memory \& Cognition, 33, 1085-1095.

Lien, M.-C., \& Proctor, R. W. (2002). Stimulus-response compatibility and psychological refractory period effects: Implications for response selection. Psychonomic Bulletin \& Review, 9, 212-238.

MudD, S. A. (1963). Spatial stereotypes of four dimensions of pure tone. Journal of Experimental Psychology, 66, 347-352.

PAshler, H. (2000). Task switching and multitask performance. In S. Monsell \& J. Driver (Eds.), Attention and performance XVIII: Control of cognitive processes (pp. 277-307). Cambridge, MA: MIT Press.

Weeks, D. J., \& Proctor, R. W. (1990). Salient-features coding in the translation between orthogonal stimulus and response dimensions. Journal of Experimental Psychology: General, 119, 355-366.

(Manuscript received June 18, 2004; revision accepted for publication May 15, 2006.) 